\title{
Updated Evaluation of IV Dihydroergotamine (DHE) for Refractory Migraine: Patient Selection and Special Considerations
}

This article was published in the following Dove Press journal:

Journal of Pain Research

\section{Rafia Shafqat \\ Yadira Flores-Montanez \\ Victoria Delbono \\ Stephanie J Nahas}

Department of Neurology, Thomas Jefferson University, Philadelphia, PA, USA
Correspondence: Stephanie J Nahas 900 Walnut St., Suite 200, Philadelphia, PA 19107-5509, USA

Tel + I 215955 1960

Fax +I 2159060335

Email stephanie.nahas@jefferson.edu

\begin{abstract}
Dihydroergotamine (DHE) is an ergot alkaloid derivative of substances produced by rye fungus. Ergotamine was first used in the field of gynecology and obstetrics, then used for migraine treatment a few years later. DHE was developed as a derivative of ergotamine. DHE, when compared to ergotamine, demonstrates greater alpha-adrenergic antagonist activity, lower arterial vasoconstriction, less dopaminergic agonism, and lower emetic potential. DHE can be delivered via several routes including intravenous (IV), intramuscular (IM), subcutaneous (SC), intranasal (IN), oral, and orally inhaled (although the latter two are not available in the USA and the last remains experimental only). DHE can be used in an outpatient basis in infusion centers, emergency departments, and urgent care centers, as well as inpatient treatment for admitted patients. There are protocols for adults as well as pediatric migraine treatment. DHE and other ergot alkaloids are considered contraindicated in pregnant women as they decrease uterine blood flow and increase uterine muscle contractility predisposing to spontaneous abortion. DHE during lactation is also not recommended as it can lead to gastrointestinal distress and weakness in infants; it can also suppress milk production. Caution should be taken before administering DHE in patients with cardiovascular risk factors. DHE is an older drug with an interesting history, yet it is still clinically useful today for patients with migraine attacks not responsive to triptans, who have a greater burden from migraine, and in refractory migraine.
\end{abstract}

Keywords: ergotamine, rye fungus, pregnant, lactating, cardiovascular risk factors, infusion, inpatient, pediatric

\section{Introduction}

Migraine is an under-recognized and undertreated neurologic condition that can be significantly costly and disabling. Migraine has been found to be the second leading cause of disability in the United States when it comes to "impaired quality of life, substantial lost productivity, and high economic costs". ${ }^{1}$ Several theories behind the etiology of migraine have been put forth over centuries, including both vascular and neuronal, until ultimately in 2002, Goadsby, Lipton, and Ferrari described migraine as a neurovascular condition. ${ }^{2}$ Treatments for migraine include preventive and acute, and in one way or another, they address certain aspects of migraine pathophysiology. With respect to acute therapy, there has been an evolution from treatments that are non-specific (ie, they target aspects not unique to migraine) to the more specific (ie, they target the unique aspects) driven by the need for more effective and better tolerated approaches. One of the first synthesized migraine- 
specific treatments is dihydroergotamine (DHE). Here we discuss the coming of age of DHE through its prototype, its pharmacology, and its pharmacokinetics, and more importantly, the special considerations - who should use it, under what circumstances, and when to use with caution.

\section{History of Dihydroergotamine}

Dihydroergotamine (DHE) is an ergot alkaloid borne from the discovery of ergots, substances produced by rye fungus and recognized to have interesting biologic properties centuries ago. The first ergot used in modern medicine is ergotamine, which was isolated from its ergot predecessor in 1918. ${ }^{3}$ Ergotamine was initially used for its uterotonic effects in the field of gynecology and obstetrics under the name Gynergen (ergotamine tartrate) in 1921. Ergotamine was first used for the treatment of migraine in 1925 by Maier in Switzerland, when it was originally postulated that migraine was caused by increased sympathetic activity and that ergotamine could reverse that effect based on its properties. Not long after, Graham and Wolff showed a parallel decrease in temporal pulsations and headache after the use of intravenous (IV) ergotamine, inspiring the Vascular Theory of Wolff. This theory stated that an initial cerebral vasoconstriction would be followed by vasodilation and could explain symptoms of migraine such as aura (linked to vasoconstriction) and throbbing pain (caused by pulsating dilated arteries). ${ }^{3,4}$ Although today this theory is no longer considered valid, the supposition was that a vasoconstrictor such as an ergot could reverse the vasodilation and thus the pain of migraine. Ergotamine was introduced to the USA in 1934 following several European studies demonstrating its successful use in migraine treatment.

It was not until 1943 that DHE was first developed as a derivative of ergotamine. DHE, when compared to ergotamine, demonstrates greater alpha-adrenergic antagonist activity, lower arterial vasoconstriction, less dopaminergic agonism, and lower emetic potential. ${ }^{5}$

DHE was largely used and studied as an acute treatment for migraine at first. It was later found that DHE, while effective in acute treatment, required higher doses for the same effects when compared to ergotamine. Because of its weaker vasoconstrictive properties, later studies supported the notion that DHE might be used as a prophylactic treatment. ${ }^{2}$ Currently, it is used in some countries under this indication, but not in the USA.
For years, ergotamine and DHE were the only migraine-specific treatment options until the era of the triptans. Now many guidelines list triptans and DHE as first-line therapies for moderate to severe migraine attacks. DHE has been found to be helpful with menstrual migraine, status migrainosus, rebound headache, and chronic daily headache. ${ }^{6}$ DHE has also been found to be effective in triptan non-responders. ${ }^{7}$

\section{Formulations}

DHE can be delivered via several different routes including IV, intramuscular (IM), subcutaneous (SC), intranasal (IN), oral, and orally inhaled (although the latter two are not available in the USA and the last remains experimental only). Pharmacokinetics, and thus efficacy and tolerability, vary with each route of administration.

Oral DHE was first formulated in 1943. It was not until much later that the IV formulation (in 1987) and the IM formulation (in 2001) of DHE were studied formally and shown to be effective in the treatment of migraine.

An extensive literature review written by Silberstein and Kori revealed the following when comparing each of the different formulations: 1) DHE, in general, is more effective than placebo but less effective than sumatriptan; 2) orally inhaled DHE likewise is more effective than placebo, though no comparative studies with sumatriptan had been done; 3) SC sumatriptan provides more rapid relief than DHE, but DHE results in less headache recurrence compared to sumatriptan; 4) among parenteral formulations of DHE (including SC, IM, IV, and IN), efficacy is superior with injectables, though adverse effects, including nausea, are worse with this dosing. ${ }^{7}$

IN dosing carries advantages in that it is a convenient method of delivery with broad patient acceptability. Nasal sprays are more accessible while in the workplace and more acceptable to those with needle phobia; thus, noninjectable formulations are important options for outpatient management.

\section{Pharmacology}

DHE is one of the derivatives of ergot alkaloids, which come from the fungus Claviceps purpurea that grows in the ears of rye. ${ }^{8}$ The chemical structure of all ergot alkaloids includes the ergoline ring. ${ }^{9}$ DHE is a semisynthetic, hydrogenated ergot alkaloid synthesized by reducing an unsaturated bond in ergotamine. ${ }^{10}$ This chemical modification results in an ergot derivative with greater alphaadrenergic antagonist activity and reduced vasoconstrictor 
and emetic potential. ${ }^{10}$ DHE is structurally similar to lysergic acid diethylamide (aka LSD); however, it does not carry hallucinogenic properties. ${ }^{8}$ DHE also has structural similarities to serotonin, norepinephrine, and dopamine, therefore its purported mechanism of action is by acting on various catecholamine receptors. ${ }^{8}$

The exact mechanism of action is not known; however, it is proposed to include inhibition of neurogenic inflammation, blockade of transmission in the trigeminal nucleus caudalis, and possibly, vasoactive effects (eg, constriction of large capacitance arteries, closure of arteriovenous anastomoses). ${ }^{10}$ It is believed that these effects are due to its potent agonist activity at $5 \mathrm{HT}-1 \mathrm{~B},-1 \mathrm{D}$, and $-1 \mathrm{~F}$ receptors. The most common adverse effects seen with DHE include nausea, vasoconstriction, and dizziness, suggesting that DHE indeed acts also on dopaminergic, muscarinic, and adrenergic receptors. ${ }^{8}$

DHE has low oral bioavailability and is subject to substantial (greater than 90\%) first-pass metabolism. ${ }^{9}$ However, the metabolites of DHE have actions similar to those of the parent drug, which leads to potentiation of effect. ${ }^{9}$ In addition, oral DHE has incomplete and inconsistent drug passage across the gastrointestinal mucosa, making it suboptimal for clinical use. $^{10}$ The absolute bioavailability of SC/IM/IV DHE is $100 \%$, and for intranasal administration, it is approximately $40 \%{ }^{8}$ Peak plasma levels occur approximately 1 to 2 minutes after IV administration, 24 minutes after IM administration, and 30 to 60 minutes after intranasal administration., Parenteral administration of DHE avoids first-pass hepatic metabolism and delivers adequate plasma concentrations of the drug. ${ }^{8,10}$ DHE is rapidly and extensively metabolized in the liver with only about $6-7 \%$ excreted in the urine. ${ }^{10}$ The major route of elimination is the feces following biliary excretion of unchanged drug and metabolites. ${ }^{10}$ DHE has many metabolites, but only the ones that contain the ergoline ring structure of ergot alkaloids are active in migraine. ${ }^{10}$ The major metabolite is 8-OH DHE, which attains a concentration 5-7 times greater than that of DHE itself, with pharmacological effects qualitatively similar to those of the parent compound. ${ }^{10}$ The elimination half-life of DHE is biphasic, with peaks at approximately 0.7 to 1 hour and then 10 to 13 hours. ${ }^{9}$

\section{Patient Selection and Clinical Use}

Guidelines in the United States and France recommend migraine-specific therapies like triptans and DHE as firstline treatment for moderate to severe attacks. ${ }^{7}$ DHE is considered generally beneficial in difficult to treat cases, for example, those with menstrual migraine, rebound headache, migraine recurrence, status migrainosus, or chronic daily headache. ${ }^{7}$ A typical intranasal dose consists of one spray $(0.5 \mathrm{mg})$ into each nostril then repeated after 15 minutes. A typical injectable dose is 0.5 to $1 \mathrm{mg}$. It is generally recommended to limit dosing to $3 \mathrm{mg}$ per 24 hours and to $6 \mathrm{mg}$ per week, though it has been established safe and effective to give up to $3 \mathrm{mg}$ per day for up to 7 days in a controlled setting.

Both triptans and DHE act on 5HT-1B and -1D receptors but have different pharmacodynamics. Both can be useful in the treatment of moderate to severe migraine attacks, with sumatriptan providing more rapid relief and DHE being more effective in reducing recurrence. ${ }^{7}$ Often DHE is reserved as an option for patients whom triptans have failed but has value also in difficult to treat headaches and those complicated with medication overuse. ${ }^{7}$

\section{Outpatient Infusion Centers}

Raskin developed the original repetitive dose IV DHE protocol to treat refractory migraine in 1986 . This became the foundation for inpatient and in-clinic treatments administered over a number of days. The original protocol called for IV DHE $0.3-1 \mathrm{mg}$ every 8 hours with IV metoclopramide $10 \mathrm{mg}$ for 2-3 days. ${ }^{11}$ Raskin's original protocol has been studied against various other treatments over the years, including IV meperidine and IV promethazine, in which it resulted in similar efficacy yet significantly fewer adverse effects, ${ }^{12}$ and IV ketorolac, in which it demonstrated a greater degree of improvement. In the outpatient infusion setting, a modification of this protocol is typically undertaken to allow for up to $2 \mathrm{mg}$ of DHE in divided doses (and other medications as necessary) to be delivered over several hours and repeated on consecutive days. Continuous intravenous DHE administration in an outpatient home-based setting is also another option for patients. ${ }^{13}$ Charles et al conducted a prospective study to treat patients with a daily intractable headache by administering intravenous DHE infusion at their home. They were administered a total of $7.5 \mathrm{mg}$ IV DHE over three consecutive days under the care of a registered nurse. ${ }^{13}$ Thirty-five patients were treated, $33 \%$ became headachefree and $64 \%$ had a reduction in their headache at the end of 3 days. ${ }^{13}$ It is a cost-effective treatment option for patients and avoids hospitalization for migraine treatment.

A favorable adverse event profile, high degree of efficacy, and lack of need for close laboratory or telemetry monitoring make treatment with IV DHE an attractive option in the outpatient setting. The benefits of outpatient 
IV DHE infusion also include less time commitment, less cost to both the patient and the healthcare system, and no exposure to noisy, brightly lit emergency departments.

\section{Emergency Departments and Urgent Care Centers}

Patients who do not have adequate at-home treatment options lack access to outpatient infusion centers, or who have intractable nausea and vomiting may present to an emergency department (ED) or urgent care center (UCC) for treatment. There have been few strong studies of DHE use in the $\mathrm{ED}^{14}$ and none in the UCC setting. In one class 3 study in the ED, patients were randomized to DHE $1 \mathrm{mg}$ SC or sumatriptan $5 \mathrm{mg} \mathrm{SC}$. The outcome favored sumatriptan at 1 and 2 hours though not at 3 hours, suggesting a more durable effect of DHE at aborting a migraine attack. Due to the lack of rigorous studies, however, the use of IV DHE in the ED setting does not garner a strong recommendation. Despite this, it is used commonly in many EDs and UCCs, often as part of a "migraine cocktail" incorporating antiemetic and antiinflammatory drugs. Further study is warranted to establish best practices with DHE under these circumstances.

\section{Inpatient}

Certain populations of patients benefit from inpatient admission for the management of migraine. Among them include patients with status migrainosus, dehydration or intractable nausea/vomiting, dependence on opioids or barbiturates requiring detoxification, medical disease requiring careful observation, outpatient treatment failure, frequent $\mathrm{ED}$ visits, and those requiring comprehensive medical or psychiatric co-management. ${ }^{16}$

For patients with a longstanding history of refractory migraine, inpatient treatment improves pain and disability and reduces analgesic overuse. ${ }^{7,16}$ Multiple drugs can be used in the inpatient setting along with DHE to enhance its effects, manage other symptoms of migraine, and mitigate adverse events. Inpatient use was first described by Raskin, in which 55 patients with continuous headache, most of whom were overusing acute medications, received IV DHE at a dose between $0.3 \mathrm{mg}$ and $1 \mathrm{mg}$ every 8 hours for 2 days. At the end of treatment, $89 \%$ of patients were headache-free with most reporting lasting benefit. ${ }^{11}$

In a publication by Silberstein and colleagues, longterm prognosis was evaluated in 50 patients admitted for inpatient management of chronic daily headache and medication overuse. They found that $87 \%$ of patients who were detoxified from acute drug overuse and treated with repetitive IV DHE continued to have an improvement for up to 2 years. ${ }^{15}$ In their experience, IV DHE is effective in rapidly controlling intractable headache, with most patients achieving improvement in 2 to 3 days. ${ }^{15}$ Other studies also support the effectiveness of IV DHE in reducing headache frequency and severity. This includes a study by Nagy et al who evaluated 114 patients with chronic migraine treated with IV DHE; $67 \%$ of patients reported headache freedom at discharge, and $75 \%$ reported headache freedom 1 month after treatment. ${ }^{17}$

Protocols of administrating DHE vary from center to center. The University of California San Francisco Headache Center administers DHE every 8 hours with an initial dose of $0.5 \mathrm{mg}$ escalated to $1 \mathrm{mg}$ as tolerated. In pediatric patients, the dose is adjusted for weight in patients under $50 \mathrm{~kg} .{ }^{18}$

Special considerations to take with IV DHE revolve around its potential adverse events and contraindications. The most common side effect is nausea. Many patients require a concomitant antiemetic. A more recent adverse event has become apparent with the increasing use of DHE in the inpatient setting in patients with difficult IV access. In a retrospective review of patients admitted to the inpatient headache unit at University of California San Francisco Headache Center, patients undergoing DHE infusion who had a mid-line or peripherally inserted central catheter (PICC line) had a higher risk of deep venous thrombosis (DVT) or pulmonary embolism (PE) than patients undergoing treatment without DHE or with only peripheral access. Of 263 admissions for IV DHE treatment given trough PICC or midline, 19 had PE, DVT, or superficial vein thrombosis. ${ }^{18}$ Of the 10 adults with DVT or $\mathrm{PE}$, all required anticoagulation; 1 pediatric patient needed anticoagulation. This suggests that the venoconstrictive effect of DHE around a longer foreign surface (PICC line or mid-line) increases the risk of thrombus formation. Given that no thrombotic effect was associated with peripheral IV access, the authors recommend the use of peripheral IV lines for administrating IV DHE whenever possible. ${ }^{18}$

Long-term success depends not only on providing aggressive IV treatment for migraine but also on optimizing preventive medication, addressing psychological comorbidities and providing adequate follow-up post hospital. ${ }^{15}$ 


\section{Considerations in Special Populations \\ Pregnant or Lactating Women}

As a group, ergots are associated with increased sympathetic activity and vasoconstriction. Use in pregnancy has been associated with decreased uterine blood flow and increased uterine muscle contractility, predisposing to spontaneous abortion. Therefore, its use in pregnancy is not recommended, ${ }^{19-21}$ and DHE and other ergot alkaloids are considered contraindicated in the USA.

There are no studies prospectively evaluating adverse events and outcomes of DHE use in pregnant women. The available data come mainly from registries and animal studies. In a pregnancy registry done in Quebec, evaluating 53 pregnant women exposed to DHE, the use of DHE in pregnancy was associated with a fourfold increase risk of prematurity. In one animal study, DHE did not demonstrate teratogenic effects, ${ }^{19}$ but in another, a dose of $617 \mathrm{mg} / \mathrm{kg}$ produced 50\% embryonic mortality in rats, and with lower doses, congenital malformations were observed including cleft palate, bilateral limb defects, and lens opacity. ${ }^{20}$

The use of DHE during lactation also is not recommended. Under Hale's lactation risk, it is classified as L4 (potentially hazardous) due to reported cases of gastrointestinal distress and weakness in infants. In addition, it can suppress milk production. ${ }^{21}$

\section{Pediatric Use}

Despite its utility, relatively little has been written about DHE in this population, and therefore, its use has been limited mostly to specialists in pediatric migraine who are familiar with its effects. Intranasal DHE has been used successfully in children and adolescents who do not respond to intranasal triptans. ${ }^{22}$ IV DHE has been shown to be effective in status migrainosus in children and adolescents. ${ }^{23-25}$ There are two inpatient dosing schedules for pediatric patients described in the literature, based on weight, age, and tolerability. A lower dose protocol calls for $0.1-0.2 \mathrm{mg} /$ dose (based on age) along with a neuroleptic (eg, metoclopramide) administered every 6 hours for a maximum of 16 doses or until headache freedom is achieved. ${ }^{22}$ A higher dose protocol (for patients with inadequate response to lower doses, or who are deemed unlikely to respond to a lower dose but likely to tolerate a higher dose) utilizes $0.5-1 \mathrm{mg} /$ dose (based on age and weight) administered with a neuroleptic (eg, prochlorperazine) every 8 hours for a maximum of 20 doses. ${ }^{22}$ Two retrospective studies of these dosing regimens revealed that $74-80 \%$ of patients have an excellent response. $^{26}$

\section{Patients with Cardiovascular Risk Factors}

Given the potential for vasoconstriction, caution should be exercised in all patients. DHE, in contrast to ergotamine and triptans, has a less vasoconstrictive effect on peripheral arteries and a more pronounced effect on the veins. Hence, IV DHE was used at one time with heparin, even in elderly patients, for the prophylactic treatment of postoperative thrombophlebitis ${ }^{7}$ and in orthostatic hypotension. ${ }^{27}$ Still, use in patients who already have a predisposition for vasoconstriction, active or unstable vascular disease, or high vascular risk burden is contraindicated. It is also worth noting that the vasoconstrictive effects of DHE are more prominent in the meningeal arteries than coronary arteries. ${ }^{28}$ Baseline EKG is often recommended prior to inpatient IV DHE administration, and a more complete vascular risk assessment is often undertaken in the predisposed based on comorbidities or age.

A common side effect of IV DHE is chest pain. In a retrospective review in the Children's Hospital of Philadelphia, 24\% of patients admitted for IV DHE developed chest pain. Among them, 20\% had EKG abnormalities, but none were deemed clinically significant, and no elevations in cardiac enzymes were seen. Most of the time therapy could be restarted safely and effectively, however precautions are still warranted when patients complain of chest pain, regardless of their risk profile, and evaluation with EKG and cardiac enzymes should be undertaken when chest pain occurs. ${ }^{29}$

\section{Summary}

DHE is an older drug with an interesting history, yet it is still clinically useful today for patients with migraine attacks not responsive to triptans, who have a greater burden from migraine, and in refractory migraine. Currently, it is administered as a liquid nasal spray or by injection, though oral formulations have been used historically, and an intranasal powder is in development. DHE can be used at home, in the clinic, and in the hospital with good results in select patients. All providers who care for patients with migraine should be familiar with its properties and precautions.

\section{Disclosure}

Dr Stephanie Nahas reports personal fees from Alder/ Lundbeck, Allergan, Amgen/Novartis, Biohaven, Eli Lilly, electroCore, Impel, Supernus, Teva, Theranica, Zosano, Springer, and Wolters-Kluwer outside the submitted work. The authors report no other conflicts of interest in this work. 


\section{References}

1. Saylor D, Steiner TJ. The global burden of headache. Semin Neurol. 2018;38(2):182-190. doi:10.1055/s-0038-1646946

2. Goadsby PJ, Lipton RB, Ferrari MD. Migraine - current understanding and treatment. $N$ Engl J Med. 2003;46(4):257-270.

3. Tfelt-Hansen PC, Koehler PJ. History of the use of ergotamine and dihydroergotamine in migraine from 1906 and onward. Cephalalgia. 2008;28(8):877-886. doi:10.1111/j.1468-2982.2008.01578.x

4. Ahn AH. On the temporal relationship between throbbing migraine pain and arterial pulse. Headache. 2010;50(9):1507-1510. doi: $10.1111 / \mathrm{j} .1526-4610.2010 .01765 . \mathrm{x}$

5. Silberstein SD. The pharmacology of ergotamine and dihydroergotamine. Headache. 1997;37(Suppl 1):S15-25.

6. Baron EP, Tepper SJ. Revisiting the role of ergots in the treatment of migraine and headache. Headache. 2010;50(8):1353-1361. doi: $10.1111 /$ j. $1526-4610.2010 .01662 . x$

7. Silberstein SD, Kori SH. Dihydroergotamine: a review of formulation approaches for the acute treatment of migraine. CNS Drugs. 2013;27 (5):385-394. doi:10.1007/s40263-013-0061-2

8. Tepper SJ. Orally inhaled dihydroergotamine: a review. Headache. 2013;53(Suppl 2):S43-S53. doi:10.1111/head.12184

9. Saper JR, Silberstein SD. Pharmacology of dihydroergotamine and evidence for efficacy and safety in migraine. Headache. 2006;46 (Suppl 4):S171-S181. doi:10.1111/j.1526-4610.2006.00601.x

10. Silberstein SD, McCrory DC. Ergotamine and dihydroergotamine: history, pharmacology and efficacy. Headache. 2003;43:144-166. doi:10.1046/j.1526-4610.2003.03034.x

11. Raskin NH. Repetitive intravenous dihydroergotamine as therapy for intractable migraine. Neurology. 1986;36(7):995-997. doi:10.1212/ WNL.36.7.995

12. Scherl ER, Wilson JF. Comparison of dihydroergotamine with metoclopramide versus meperidine with promethazine in the treatment of acute migraine. Headache. 1995;35(5):256-259. doi:10.1111/j.15264610.1995.hed3505256.x

13. Charles JA, Von Dohln P. Outpatient home-based continuous intravenous dihydroergotamine therapy for intractable migraine. Headache. 2010;50:852-860. doi:10.1111/j.1526-4610.2010.01622.x

14. Orr SL, Friedman BW, Christie S, et al. Management of adults with acute migraine in the emergency department: the American headache society evidence assessment of parenteral pharmacotherapies. Headache. 2016;56:911-940. doi:10.1111/head.12835

15. Silberstein SD, Silberstein JR. Chronic daily headache: long term prognosis following inpatient treatment with repetitive IV DHE. Headache. 1992;32(9):439-445. doi:10.1111/j.1526-4610.1992.hed3209439.x

16. Marmura MJ, Goldberg SW. Inpatient management of migraine. Dep Neurol Fac Paper. 2015;Paper:101.
17. Nagy AJ, Gandhi S, Bhola R, Goadsby PJ. Intravenous dihydroergotamine for inpatient management of refractory primary headaches. Neurology. 2011;77(20):1827-1832. doi:10.1212/WNL.0b013e3182377dbb

18. Tso AR, Patniyot IR, Gelfand AA, Goadsby PJ. Increase rate of venous thrombosis may be associated with inpatient dihydroergotamine treatment. Neurology. 2017;89:279-283. doi:10.1212/ WNL.0000000000004108

19. Berard A, Kori S. Dihydroergotamine (DHE) use during gestation and the risk of adverse pregnancy outcomes. Headache. 2012;52 (7):1085-1093. doi:10.1111/j.1526-4610.2012.02172.x

20. Silberstein SD. Headaches and women: treatment if the pregnant and lactating migraineur. Headache. 1993;33:533-540. doi:10.1111/ j.1526-4610.1993.hed3310533.x

21. Burch R. Epidemiology and treatment of menstrual migraine and migraine during pregnancy and lactation: a narrative review. Headache. 2019;60:1-17.

22. O’Brien HL, Kabbouche MA, Kacperski J, et al. Treatment of pediatric migraine. Curr Treat Opt Neurol. 2015;17(1):1-17. doi:10.1007/ s11940-014-0326-1

23. Connelly M, Sekhon S, Stephens D, et al. Enhancing outpatient dihydroergotamine infusion with interdisciplinary care to treat refractory pediatric migraine: preliminary outcomes from the comprehensive aggressive migraine protocol ("CAMP"). Headache. 2019;60:1-9.

24. Kabbouche MA, Powers SW, Segers A, et al. Inpatient treatment of status migraine with dihydroergotamine in child and adolescents. Headache. 2009;49(1):106-109. doi:10.1111/j.15264610.2008.01293.x

25. Nelson GR, Bale JF, Kerr LM. Outcome and cost of inpatient hospitalization for intravenous dihydroergotamine treatment of refractory pediatric headache. Pediatr Neurol. 2017;66:76-81. doi:10.1016/j. pediatrneurol.2016.09.009

26. Patniyot IR, Gelfand AA. Acute treatment therapies for pediatric migraine: A qualitative systematic review. Headache. 2016;56 (1):49-70. doi:10.1111/head.12746

27. Horbach T, Wolf H, Michaelis HC, et al. A fixed-dose combination of low molecular weight heparin with dihydroergotamine versus adjusted dose unfractionated heparin in the prevention of deep-vein thrombosis after total hip replacement. Thromb Hemost. 1996;75 (2):246-250. doi:10.1055/s-0038-1650253

28. Labruijere S, Chan KY, de Vries R, et al. Dihydroergotamine and sumatriptan in isolated human coronary artery, middle meningeal artery and saphenous vein. Cephalalgia. 2015;35(2):182-189. doi: $10.1177 / 0333102414544977$

29. Fridinger S, Szperka C. the cardiovascular impact of intravenous dihydroergotamine for pediatric headache patients. Neurol Rev. 2018;page 6.
Journal of Pain Research

\section{Publish your work in this journal}

The Journal of Pain Research is an international, peer reviewed, open access, online journal that welcomes laboratory and clinical findings in the fields of pain research and the prevention and management of pain. Original research, reviews, symposium reports, hypothesis formation and commentaries are all considered for publication. The manuscript management system is completely online and includes a very quick and fair peer-review system, which is all easy to use. Visit http:// www.dovepress.com/testimonials.php to read real quotes from published authors. 